\title{
Zeitgenössische deutsche Reflexionen über die Rolle des Faktors Arbeit in den frühen Phasen der britischen Industrialisierung (1750-1850)
}

Teuteberg, Hans Jürgen

First published in:

Wirtschaftspolitik und Arbeitsmarkt, S. 238 - 270, Wien 1974

Münstersches Informations- und Archivsystem multimedialer Inhalte (MIAMI) URN: urn:nbn:de:hbz:6-66459441493 
Hans J. Teuteberg

ZEITGENOSSISCHE DEUTSCHE REFLEXIONEN UBER DIE ROLLE DES FAKTORS ARBEIT IN DEN FRUUHEN PHASEN DER BRITISCHEN INDUSTRIALISIERUNG (1750-1850)

Seit mehr als einhundert Jahren gehört die "Soziale Frage“ als Pendant des ökonomisch-technischen Industrialisierungsgeschehens zum Kanon sozialwissenschaftlicher wie kulturkritisch-historischer Diskussion. In diesem Rahmen fand die frühindustrielle Lohnarbeiterschaft in Großbritannien deutscherseits oft ein spezielles Interesse, wurde doch ihre Genesis vielfach als ein soziales Lehrmodell interpretiert, aus dem sich universale Theorien über die Bildung der sozialen Klassen und daraus folgend über die Entwicklung wirtschaftlicher und gesellschaftlicher Systeme ableiten lassen. Das Buch „Die Lage der arbeitenden Klasse in England“ von Friedrich Engels, das dieser nach einem zweijährigen Aufenthalt in Manchester innerhalb weniger Monate konzipierte und im März 1845 veröffentlichte, ist bis heute die klassische Aufzeichnung darüber geblieben ${ }^{1}$. Nicht nur Karl Marx und seine Epigonen, auch die bürgerliche Wissenschaft hat immer wieder darauf zurückgegriffen ${ }^{2}$. Noch heute wird das Werk des damals 24jährigen

1 F. Engels, Die Lage der arbeitenden Klasse in England. Nach eigener Anschauung und authentischen Quellen, 1845, 21892, 31909, 41921. Hist.krit. Nachdruck der Originalfassung von 1845 in: K. Marx und F. Engels, Historisch-kritische Gesamtausgabe. Werke/Schriften/Briefe, hrsg. von D. Rjazanow und V.Adoratskij, 1/4, 1932, 5-282; neueste Auflagen der Ausgabe letzter Hand in der Bücherei des Marxismus-Leninismus 30, 1964, und in der vom Verlag J.H. Dietz Nachfolger herausgegebenen Reihe Sozialistische Klassiker in Nachdrucken, 1965; erste englische Fassung: The Condition of the Working-Class in England in 1844. Translated by F. K. Wischnewetzky, 1887 und 1892 (wissenschaftlich unbrauchbar); hist.-krit. Edition und Neuübersetzung durch W. O. Henderson und W. H. Chaloner, 1958, 21965. Auszug: F. Engels, Selected Writings. Edited and introduced by W. O. Henderson, (Penguin Books A 729), 1967.

2 Bekannteste Beispiele aus dem Lager der bürgerlichen Wissenschaft sind etwa W. Sombart, Die gewerbliche Arbeiterfrage, 1904; H. Herkner, Die Arbeiterfrage, 51908 ; G. M. Trevelyan, Kultur- und Sozialgeschichte
Barmer Fabrikantensohns hinsichtlich der von ihm geschilderten Arbeitsverhältnisse vielfach unbesehen akzeptiert, wenngleich seine Prognosen - von der empirischen Wirtschafts- und Sozialforschung schon im späten 19. Jahrhundert widerlegt - nicht mehr zur akademischen Debatte anstehen. Der Grund für das eigentümlich starre Festhalten an den deskriptiv-analytischen Teilen des Buches dürfte nicht nur mit ideologischen Motiven, sondern auch mit der Tatsache zusammenhängen, daß das Problem der Frühindustrialisierung insgesamt noch zuwenig erforscht ist. Bekanntlich ist es erst in jüngster Zeit ins Zentrum historischen Bemühens gerückt ${ }^{3}$. In diesem Zusammenhang wird es nun Zeit, die immer wieder stereotyp tradierten Engelschen Aussagen im Licht moderner Forschungen erneut zu überprüfen.

Unerklärlicherweise ist man bisher noch nicht auf die Idee gekommen, die Berichte anderer deutscher Zeitgenossen einmal systematisch auszuwerten, die die Anfänge des britischen Industriestaates ebenso aufmerksam und meistens sogar an Ort und Stelle miterlebten. Der Zweck der nachfolgenden Ausführungen ist es, einige dieser Stimmen erstmals zu Wort kommen zu lassen. Die "Soziale Frage" wird damit bewußt nicht anhand bekannter klassischer Gesellschaftstheorien, sondern in konsequenter Rückkehr ad fontes noch einmal aus dem Mund der damals lebenden deutschen Zeitgenossen erklärt. Mit der akribischen Aufnahme damaliger Berichte, Fragestellungen und Kommentare, wie sie aus der direkten Anschauung entstanden sind, können vor allem einige Standardthesen über die Arbeitsmarktsituation in der Frühindustrialisierung neu überdacht werden. Die Befragung der zeitgenössischen Autoren zwischen 1750 und 1850 hat dabei einen kaum zu überschätzenden Vorteil: Die Schilderungen sind noch völlig unberührt von den großen ideologischen Kontroversen des späten 19. Jahrhunderts - entscheidende Probleme werden noch ganz unbelastet von den großen Theorien dargestellt. Einige der Autoren sind gleichsam Arm in Arm mit Friedrich Engels durch

Englands, 1948. Diese Liste von Autoren und Titeln läßt sich natürlich noch weit verlängern.

$3 \mathrm{Vgl}$. O. Büsch, Industrialisierung und Geschichtswissenschaft, 1969. W. Fischer, Okonomische und soziologische Aspekte der frühen Industrialisierung, in: ders. (Hrsg.), Wirtschafts- und sozialgeschichtliche Probleme der frühen Industrialisierung, 1968, 1-20. 
Manchester gewandert und haben sich von ihm die Schattenseiten der heraufziehenden neuen Industriegesellschaft erklären lassen. Mit dem Rückgriff auf diese Quellen wird ein Nachteil vermieden, der einer bestimmten späteren Literatur immer wieder zum Vorwurf gemacht werden kann: Daß sie nämlich wirtschaftlich-gesellschaftliche Streitfragen der eigenen Zeit undifferenziert in frühere Epochen hineinzieht, wo diese in dieser Form noch gar nicht bestanden haben.

Natürlich ist es bei einem knappen Abriß nicht möglich, die deutschen'Englandautoren und ihre Anglicana näher zu charakterisieren oder auf die deutschen und englischen Vorarbeiten, geschweige denn auf andere Quellen zu dieser Problemstellung näher einzugehen. Für den vorliegenden Zweck konnten aus Hunderten von Englandberichten auch nur einige ganz wenige Aspekte paradigmatisch ausgewählt werden: Untersucht wird im ersten Teil die Entwicklung des Arbeitskräfteangebots, genauer gesagt die Herkunft der industriellen Lohnarbeiterschaft oder nach einer berühmten Marxschen Formel die „Rekrutierung der industriellen Reservearmee", wobei die vielbesprochene und oftmals mißverstandene Umschichtung der Arbeitskräfte vom primären agrarischen zum sekundären industriellen bzw. tertiären dienstleistenden Sektor im Mittelpunkt steht ${ }^{4}$. Der zweite Teil widmet sich dann dem Wandel der Arbeitsverfassung und greift als Beispiel das Problem der Kinderarbeit heraus. Viele mit der Klärung der Arbeitsmarktlage ebenfalls zusammenhängende Fragen müssen unberücksichtigt bleiben: Die Lohn- und Lebensstandardproblematik, soziale Bewegung, Armen- und Fabrikgesetzgebung sowie die zeitgenössischen Stimmen über die veränderte Stellung des „Proletairs" in der Gesamtgesellschaft. Auch Bevölkerungsvermehrung und Verstädterung sowie das Verhältnis des frühindustriellen Arbeiters zur Technik, zur außerbetrieblichen Umwelt, zur Ge-

${ }^{4}$ Die Einteilung der Volkswirtschaft in einen primären, sekundären und tertiären Sektor ist zunächst mit dem Namen des aušrralischen Ökonomen Colin Clark verbunden gewesen und frühzeitig auch von der deutschen Literatur aufgegriffen worden. Eine wirtschaftshistorische Stufentheorie gestaltete daraus aber erst J. Fourastié, Le Grand Espoire $\mathrm{du} \mathrm{XXe}$ Siècle. Progrès technique-progrès économique-progrès social, 1949. Deutsch: Die große Hoffnung des 20. Jahrhunderts, ${ }^{2} 1969$. Vgl. audh H. Freyer, Theorie des gegenwärtigen Zeitalters, 1956, der den Begriff des „sekundären Systems“ daraus herleitete. sundheit und Hygiene, zur Kriminalität, Bildung und Religion können höchstens gestreift werden. Im Mittelpunkt soll hier einzig und allein die Frage stehen: Wie elastisch hat der Arbeitsmarkt in den frühen Stadien der britischen Industrialisierung auf die aus den ökonomisch-technischen Wandlungen herrührenden neuen Anforderungen reagiert?

Das Interesse am "niederen Volk" ist bei den deutschen Englandreisenden, die zumeist dem Bildungs- und Besitzbürgertum oder dem Adel entstammten, bis zur Französischen Revolution noch nicht groß gewesen. Zwar war die Wildheit und Roheit des englischen „Pöbels“ mit seinem sonderbaren Fremdenhaß schon frühzeitig aufgefallen; aber das war kein Grund, sich deswegen näher damit zu beschäftigen. Nach offenbar weitverbreiteter Ansicht repräsentierte der "Pöbel“ nicht das Volk an sich: er war vielmehr allerorts dumm, roh, verderbt und tölpelhaft ${ }^{5}$. Erst unter dem Einfluß der Schriften von Montesquieu, Voltaire und Rousseau, aber auch von Hume, Ferguson, Smith, Burke und Malthus samt ihren deutschen Interpreten begann man, die sozialen Unterschichten in England näher zu studieren. Die ersten Berichte, bei

5 Vgl. z. B. J. W. von Archenholtz, England und Italien 3, 21787, 117; G. F. A. Wendeborn, Der Zustand des Staates, der Religion, der Gelehrsamkeit und der Kunst in Großbritannien gegen Ende des 18. Jahrhunderts 2, 1784, 255; H.M.F. von Watzdorf, Briefe zur Charakteristik von England gehörig, geschrieben auf einer Reise im Jahre 1784, 1786, 48; (J. G. B. Büschel), Neue Reisen eines Deutschen nach und in England im Jahre 1783, 1784, 44. Ein anderer deutscher Englandbeobachter wollte dem englischen Pöbel überhaupt alle nationalen Wesenszüge absprechen und wies auf die Ubiquität des „Pöbels“ hin, der sich an allen Orten nur zu fatal gleiche. Damit wollte er allerdings noch nicht auf ein gemeinsames Klassenbewußtsein oder eine internationale Solidarität des Pöbels aufmerksam machen, sondern auf die Tatsache, daß der Mensch in seinen beiden extremen Daseinslagen höchster und niederster Kultur jene scharfen Züge verliere, die man als nationale Individualität bezeichne. Menschen könnten einerseits durch vielseitige Bildung einen so kosmopolitischen und allgemeinen Charakter annehmen, daß sich die angeborenen spezifischen nationalen Wesenszüge verwischen; sie könnten aber auch andererseits bei moralisch-physischer Verwilderung ihren Nationalcharakter verlieren. Der „Pöbel“ nähere sich der Stufe der Tiere, und diese seien bekanntlich überall gleich. Vgl. C. A. G. Goede, England, Wales, Irland und Schottland. Erinnerungen an Natur und Kunst aus einer Reise in den Jahren 1802 und 1803; 1804, 358.

16 Kellenbenz, Wirtschaftspolitik 
denen Justus Mösers Schilderung einer Bettlerkneipe im Londoner Armenviertel St. Giles und die des Pastors der deutschen Gemeinde in London hervorragen, beschäftigten sich zunächst mit der städtischen Armut ${ }^{6}$. Ubereinstimmend wurde der Luxus des "gemeinen Mannes" kritisiert: Während der deutsche Handwerker sich von „Leuten vom Stand" schon in der Kleidung unterscheide, will in den Städten Englands jedermann feine Kleidung tragen, und „wer sich nicht neue anschaffen kann, der kauft abgetragene aus zwoter Hand, damit er wenigstens schäbig vornehm aussehe "7. Die Menge der arm Geborenen und später Verarmten ist nach Angaben des deutschen Pastors, der 20 Jahre in London lebte und ein mehrbändiges repräsentatives Englandwerk verfaßte, „unglaublich groß“. Aber dafür hatte man wenig Mitgefühl: Arme waren, wie Möser formulierte, eine Gruppe von Menschen, die sich selbst vom öffentlichen Leben ausgeschlossen hatten. Da Arme dem Gemeinwesen zur Last fielen, mußte alles getan werden, um die Armut einzuschränken, die ihre Hauptursache im "Müßiggang" hatte. Armut hatte daher etwas Verächtliches ${ }^{8}$. Ein Zusammenhang mit einer wie immer gearteten "Arbeiterfrage“ wurde deutscherseits vor der Französischen Revolution natürlich noch nicht gesehen. Ganz im Gegenteil glaubte Möser, in der Nähe von „Industrieanstalten" und „Fabriken" die wenigsten Bettler zu bemerken. Was deutsche Besucher erschreckte, war auch nicht der Tatbestand der Armut an sich, sondern die massenhafte Zusammenballung von Pauperen an einem Ort. Friedrich Cottas langjähriger Englandkorrespondent Johann Christian Hüttner hat in vielen deutschen

B J. Möser, Patriotische Phantasien ("Das Glück der Bettler"), in: Sämtliche Werke, hist.-krit. Ausgabe, bearbeitet von L. Schirmeyer 4 1943, 65-68; Wendeborn, Der Zustand des Staats 1, 149-155. Vgl. ferner R. Potter, Observations on the Poor Law, on the Present State of the Poor, and Houses of Industry, 1775; H. Mayhew, London Labour and the Poor, 3 vols., 1851-1852; G. Nicholls, A History of the English Poor Law, 2 vols., ${ }^{2} 1904$; A. W. Coats, Economic Thought and Poor Law Policy in the 18th Century, EHR 2nd ser. 8, 1960-1961; D. Marshall, The English Poor in the 18th Century: A Study in Social and Administrative History, 1926.

7 Wendeborn, Der Zustand des Staats 1, 151.

8 J.Möser, Etwas zur Verbesserung der Armenanstalten. Sämtliche Werke 4, 69; ders., Ökonomische Aufgabe der Armen betreffend. Sämtliche Werke 9, 1954, 59-60.
Journalen um 1800 die unbekannte Welt des Londoner „Pöbels“ mit allen seinen Abnormitäten und Kuriositäten geschildert ${ }^{9}$. Seitdem gehörte das englische "Lumpenproletariat" zu den Standardthemen der deutschen Englandliteratur. Immer wieder beschrieben deutsche Reiseschriftsteller, bei denen Heinrich Heine nur einer von vielen ist, die „abscheulichen Quartiere des Elends", wobei man fast niemals vergaß, das "Paradies der Reichen" dem „Höllenpfuhl des Lasters, der Not und menschlicher Verderbnis“ gegenüberzustellen. Für die meisten war das städtische Massenelend zugleich auch das Gesicht der modernen Großstadt, die im kleinstädtischen Deutschland noch unbekannt war. Auch anderen kontinentaleuropäischen Besuchern ist frühzeitig der besonders krasse Gegensatz zwischen arm und reich auf der Insel aufgefallen ${ }^{10}$. Seit dem Ende der sechziger Jahre des 18. Jahrhunderts

9 (J. C. Hüttner), Sittengemählde (sic!) von London. Nebst einer vergleichenden Charakteristik seiner Bewohner, 1801, 155-162. - Vgl. ferner Hüttners regelmäßig erschienenen Englandartikel in: „Der Neue Teutsche Merkur" (hrsg. von C. M. Wieland, 1798-1800), in: „Englische Miscellen“ (hrsg. von J. F. Cotta, 25 Bde., 1800-1807), und in: „London und Paris" (hrsg. von C. A. Böttiger, 30 Bde., 1798-1815, mit Unterbrechungen und wechselndem Titel erschienen). Uber das Londoner "Lumpenproletariat" berichten u. a. ferner J. C. Fabricius, Briefe aus London vermischten Inhalts, 1784, 243 f.; F. W. Schütz, Briefe über London, 1792, 17-18; Goede, England 2, 358-388; T. F. M. Richter, Bemerkungen über England und Reise nach Sicilien, in: ders., Reisen zu Wasser und zu Lande in den Jahren 1805 bis 1817, 6, 31850, 80-84; J. W. J. Bornemann, Einblicke in England und London im Jahre 1818, 1819, 125 bis 130; H. Meidinger, Reisen durch Großbritannien und Irland vorzüglich in topographischer, kommerzieller und statistischer Hinsicht 1, 1828, 52; H. Heine, Englische Fragmente. Sämtliche Werke, hrsg. von A. Strodtmann 3, 1861, 22-24; C. Ludovic, Flüchtige Bemerkungen auf flüchtiger Reise durch einen Theil von Belgien, Holland und England, 1846, 231; F. Lewald, England und Schottland 1, 1851, 309-311; E. von Merck, Reise-Erinnerungen aus London und Paris. 2. Abdruck, 1852, 5; M. von Meysenbug, Memoiren einer Idealistin 2, 1876, 132-134.

${ }_{10}$ So hat z. B. der zwischen 1751 und 1789 in London lebende italienische Theaterdirektor, Ubersetzer und Schriftsteller Giuseppe Baretti (1719-1789) in seiner Schrift "Costumi degli Inglesi“, in den Briefen an seine Familie sowie in seiner Zeitschrift "Frusta letteraria" schon kurz nach dem Siebenjährigen Krieg als einer der ersten Ausländer auf das neue Phänomen großstädtischer Massenverelendung aufmerksam gemacht. Vgl. G. Baretti, Lettere famigliari a suoi tre fratelli Filippo, Giovanni e Amadeo, 2 Bde., 1762-1763; ders., Reise von London nach Genua durch 
gibt es Thesen über die dichotomische Aufspaltung der englischen Gesellschaft in zwei feindliche Klassen und Prophezeiungen über eine bevorstehende soziale Revolution ${ }^{11}$. Engels hat später hier eine alte Denklinie der Englandliteratur aufgegriffen. Die städtische Massenarmut vor und bei Beginn der englischen Industrialisierung wurde deutscherseits mit dem Mangel an Schulen und einer tatkräftigen Verwaltung, insbesondere dem Fehlen von Stadttoren und einer Polizei, aber auch mit der urbanen Agglomeration in Verbindung gebracht. Mehrmals wurde die 1796 erschienene Untersuchung des späteren Hamburger Generalkonsuls Patrick Colquhoun zitiert, wonach jeden Morgen in London 20.000 Menschen aufstehen, die noch nicht wissen, wie sie sich den kommenden Tag über erhalten sollen ${ }^{12}$. Nach seinem auch ins Deutsche übersetzten Buch gab es um 1800 allein in der britischen Hauptstadt 50.000 Prostituierte, 5000 Kneipenwirte und 10.000 professionelle Diebe, nicht eingerechnet das Heer der Hehler, Geldfälscher, Glücksspieler, betrügerischen Trödler, Strandräuber, Bettler, Gelegenheitslastenträger, fahrenden Musikanten und Schausteller. Die Zahl dieser am Rand der societas civilis lebenden Gruppen wurde

England, Portugal, Spanien und Frankreich. Aus dem Englischen, 1772; ders., La Frusta Letteraria. Hrsg. von L. Piccioni, 2 Bde., 1932.

${ }_{11}$ H. P. Sturz, Briefe, im Jahre 1768 auf einer Reise im Gefolge des Königs von Dänemark geschrieben, in: ders., Schriften (hrsg. von Gramberg und Merck), 1. Sammlung, 1779, 42-45; J. G. Herder, Journal meiner Reise im Jahre 1769. Hrsg. und mit einer Einleitung versehen von J. Nohl, 1949, 165; Watzdorf, Briefe, 149-154; F. Schlegel, Philosophische Vorlesungen (1800-1807), 2. Theil. Mit Einleitung und Kommentar hrsg. von J.-J. Anstett, in: Kritische Friedrich-Schlegel-Ausgabe. Hrsg, von E. Behler u. a. 13, 1964, 155-156; ders., Geschichte der alten und neuen Literatur. Vorlesungen gehalten zu Wien im Jahre 1812. Hrsg. und eingeleitet von H. Fichner, in: ebd. 16, 1961, 343; E. M. Arndt, Geist der Zeit, 1806, 328-335; nach dem Ende der napoleonischen Kriege schwoll die Zahl der deutschen wie englischen Stimmen, die eine bevorstehende soziale Revolution in England prophezeiten, noch mehr an. Aus Raumgründen können diese Titel aber hier nicht zitiert werden.

12 P. Colquhoun, A Treatise on the Police of the Metropolis, explaining the various Crimes and Misdemeanours which at Present are felt upon the Community; and suggesting Remedies for their Prevention by a Magistrate, 1796; Deutsch: Uber London's Polizey besonders in Bezug auf Verbesserungen und Verhütungsmittel der Verbrechen... Aus dem Englischen nach der 5. Aufl. übersetzt und mit einigen Erläuterungen versehen von J. J. Volkmann, 2 Theile in einem Band, 1800-1801. von Colquhoun auf 115.000 Menschen bei knapp einer Million Einwohner Londons geschätzt ${ }^{13}$.

Nur wenige bemerkten, daß es auch „ehrbare Pöbelklassen“ gab, die den Sonntag nach englischer Art feierten, ihre Wohnungen peinlich säuberten und reine Wäsche anlegten. $\mathrm{Zu}$ ihnen gehörten vor allem die Dienstboten. Sie erhielten dasselbe Essen wie die Herrschaft und waren äußerlich kaum von „Leuten vom Stand“ zu unterscheiden, was deutsche Besucher oftmals in Verlegenheit versetzte $^{14}$. Bediente wurden ebenso wie die „Kaufmannsdiener“ oder "Handlungsgehilfen“ von einem besonderen Bureau gegen eine Gebühr schon Ende des 18. Jahrhunderts vermittelt ${ }^{15}$. Außerdem war eine besondere Bedientensteuer zu entrichten. Wurde diese einmal erhöht, dann bedeutete dies für manchen eine fristlose Ent-

13 Ebd. Für ganz England beziffert Colquhoun um 1800 den "Pöbel" auf 1,320.716 Menschen, wobei aber die widersprüchlichsten Gruppen mitgezählt sind. Diese reichen vom fahrenden Volk bis zu unbescholtenen Empfängern von Armenunterstützung, von Hausierern bis $\mathrm{zu}$ temporären Erwerbslosen. Auch Dirnen und in wilder Ehe lebende Frauen werden in einen Topf geworfen. Die Schätzwerte sind daher mit großer Skepsis zu betrachten. Nach einer anderen zeitgenössischen deutschen Notiz rechnete man 1803 auf 100 Londoner Einwohner rund acht und 1820 rund elf Arme, die öffentliche Unterstützung bezogen. 1815 soll es in London 15.000 Bettler gegeben haben, wovon die Hälfte Kinder waren. 1847 wird in sechs Armenbezirken Londons, das sich natürlich schon stark auf Kosten umliegender Grafschaften vergrößert hatte, die Zahl der außerhalb von Armenhäusern unterstützten Personen mit 173.815 angegeben. Die Times vom 2. Juni 1849 berechnete die Zahl der Insassen von 24 Armenhäusern in London mit rund 15.000, womit aber nicht alle Anstalten erfaßt waren. Auch diese Zahlen sind angesichts des Fehlens genauer Statistiken dubios. Vgl. C. Otto, Reise durch die Schweiz, Italien, Frankreich, Großbritannien und Holland mit besonderer Rücksicht auf Spitäler, Heilmethoden und den übrigen medicinischen Zustand dieser Länder 2, 1825, 243-244; H. Meidinger, Das Britische Reich in Eurppa. Statistische Darstellung seiner Entwicklung, besonders in seinem jetzigen Verwaltungssystem, 1851, 576-577; E. P. Thompson, The Making of the English Working Class, ${ }^{2} 1968,59-60$.

${ }^{14}$ Schütz, Briefe über London, $91 \mathrm{ff}$. und 204; S. von La Roche, Tagebuch einer Reise durch Holland und England von der Verfasserin von Rosaliens Briefen, 1788, 404; (Büschel), Neue Reisen, 228; Wendeborn, Der Zustand des Staats 2, 246; Goede, England 2, 355-356; (J. V.) Adrian, Bilder aus England 1, 1827, 130-131; J. J. Hecht, The Domestic Servant Class in the 18th Century England, 1956.

15 Archenholtz, England und Italien 3, 34-35. 
lassung. Um 1800 soll es allein in London über 20.000 arbeitslose Dienstboten gegeben haben, die so den Angebotsdruck auf dem Arbeitsmarkt verstärkten. Wie diese Skizze zeigt, hat es schon vor und beim Beginn der Industrialisierung einen städtischen Pauperismus gegeben, der zweifellos nicht durch diese induziert worden ist. Vor allem gilt das für London, das eine reine Metropole des Handels und Handwerks war und blieb. Engels hat das nicht richtig erkannt, als er die vorindustrielle städtische Massenarmut undifferenziert mit dem Begriff des industriellen Lohnarbeiters vermengte ${ }^{* 6}$.

Neben dem "Póbel" in der Stadt war das Landvolk der Teil der Unterschichten, mit dem der deutsche Englandreisende nach seiner Ankunft zuerst in Berührung geriet. Nachwirkungen der im Humanismus aufgekommenen und im 18. Jahrhundert ins Süßliche abgebogenen Schäferpoesie mit ihrer Idyllisierung des Landlebens wie auch die Anglomanie standen aber einer realen Beobachtung zunächst im Wege ${ }^{17}$. Die ersten Berichte über die englische Landwirtschaft sind daher wenig inhaltsreich und zum Teil sogar recht schief, weil die notwendigen „data " zur Beurteilung der Zusammenhänge fehlen, wie man selbst zugibt ${ }^{18}$. Erst etwa um 1780 wurde deutscherseits erstmals klar, daß es auf der Insel gar keinen

16 Engels, Die Lage der arbeitenden Klasse in England, 1964, 85 ff. (Kap. Das industrielle Proletariat) und $89 \mathrm{ff}$. (Kap. Die großen Städte).

${ }_{17} \mathrm{Zu}$ erinnern ist hier vor allem an Sir Philipp Sidneys elisabethanischen Roman "Arcadia" (1578-1579, erweiterte Fassung 1593), der 1629 durch die Ubersetzung von Martin Opitz nach Deutschland gelangte. Seitdem drang eine Fülle von Schäferidyllen von England auf den Kontinent und formte so die Vorstellungen vom englischen Landleben. Erst George Grabbe (1754-1832), „the Poet of the Poor", schilderte die sozialen Verhältnisse auf dem Lande, wie sie wirklich waren. In seinem Werk „The Parish Register" (1807) und anderen Verserzählungen stellte er erstmals den dörflichen Alltag, wie er ihn als Landpfarrer erlebte, dem süßlichen Dorfidyll eines Oliver Goldsmith (vgl. dessen Werk "The Village" von 1783 ) gegenüber.

18 (J.L. Oeder), Beyträge zur Oekonomie, Kameral- und Polizeywissenschaft aus den Berichten eines deutschen Kameralisten von seinen Reisen nach der Schweiz, Frankreich, Holland und England im Jahre 1759 und 1763. Hrsg. von H. J. Hinze, 1782, 179 ff.; Genaue Beschreibung der Landwirthschaft auf einem Landgute in Suffolk, in: J. Beckmann, Beyträge zur Oekonomie, Technologie, Polizey - und Cameralwissenschaft 1, 1779, 48 ff.; (Büschel), Reisen, 243; Watzdorf, Briefe, 125 und 188.
"Bauernstand" in grund- und gutsherrlicher Abhängigkeit wie in Deutschland, sondern nur eine dünne Oberschicht großer und größter Landeigentümer, wenige Frei- und Erbzinsbauern sowie eine Masse von Pächtern verschiedenster Größenklassen und schließlich von diesem wiederum abhängige Landarbeiter (Field Labourer) gab ${ }^{19}$. Letztere Gruppe reichte vom qualifizierten Facharbeiter (Dreschmaschinenaufseher, Brunnen- und Drainagebauer) und dem auf Musterfarmen fest angestellten Feldarbeiter über die mit Zeitkontrakten beschäftigten Arbeitskräfte bis zu den im Tages- oder Stücklohn stehenden Aushilfsarbeitern mit deren Frauen und Kindern ${ }^{20}$.

Der deutsch-schottische Naturforscher Johann Reinhold Forster hat zwischen 1780 und 1783 in einer mittelenglischen Landstadt lebend erstmals auf deutscher Seite den Prozeß der Einhegungen

19 (J. R. Forster), Tableau de l'Angleterre pour l'année 1780, continue par l'éditeur jusqu'à l'année 1783,1784, 172; (C. G. Küttner), Beyträge zur Kenntniß vorzüglich des Innern von England und seiner Einwohner. Aus den Briefen eines Freundes gezogen von dem Herausgeber 2, 1792, 66-92; K. F. Stäudlin, Bemerkungen über Devonshire und die Insel Londy (Bruchstücke aus einer Reisebeschreibung von den Jahren 1788 , 1789 und 1790), Berlinische Monatsschrift 19, 1792, 132 f.; G. F. A. Wendeborn, Reise durch einige [der] westlichen und südlichen Provinzen Englands 1, 1793, 109-111, und 2, 23-28; F. W., Einige gelegentliche Bemerkungen über den englischen Landbau, Blätter für Polizei und Kultur 2, 1801, 40-46

20 Diese aus den Quellen entwickelte Sozialklassifzzierung wird im einzelnen interpretiert bei Thompson, English Working Class, 235; zum englischen Landarbeiter vgl. ferner J. Hasbach, Der englische Landarbeiter in den letzten hundert Jahken, Schriften des Vereins für Socialpolitik 59, 1894 (Englisch: History of the English agricultural Labourer 1908); J. L. und B. Hammond, The Village Labourer 1760-1832, 2 vols., ${ }^{4} 1948$; A. Redford, Labour Migration in England, 1800-1850, 1926; G. E. Fussel, Village Life in the 18th Century, 1947; ders., The English Rural Labourer, 1947; G.E. und F.R. Fussel, The English Country-Woman: A Farmhouse Social History, 1500-1900, 1953; E. W. Martin, The Secret People: English Village Life after 1750, 1954; F. M. L. Thompson, English Landed Society in the Nineteenth Century, 1963; C. Shrimpton, The Landed Society and Farming Community of Essex in the late 18th and early 19th Century, Phil. Diss. Cambridge, 1965; J.D. Chambers, G.E. Mingay, The Agricultural Revolution, 1750-1880, 1966; J.O. Pryce, The Problem of Rural Depopulation of Highland Britain, Wales M. A. 1967; R. Bayne-Powell, English Country Life in the Eighteenth Century (Reprint), 1937. 
in seinen ökonomischen Ursachen wie sozialen Folgen erkannt ${ }^{21}$. Bemerkenswerterweise machte er die an das System der englischen Kornzölle gekoppelten Getreideausfuhrprämien des Staates in erster Linie für die Verdrängung des Kleinpächters verantwortlich. Nach einer späteren deutschen Statistik schmolz die Zahl der Bodeneigentümer zwischen 1776 und 1816 innerhalb einer Generation auf ein Achtel zusammen22. Forster wies auch erstmals auf das Steigen der Armensteuern infolge der Einhegungen hin ${ }^{23}$. Nach seiner Schätzung ging um 1780 der zwanzigste Teil der englischen Nation am Bettelstab. Nach dem Ausbruch des englisch-franzö-

21 So schrieb Forster wörtlich: „Seit einigen Jahren ist hier eine schlechte OKonomie in England eingerissen, die das Land entvölkert und viele zur Auswanderung zwingt. Die Gutsherren übergeben ihre Ländereien Pächtern, von denen die meisten ungefähr fünfzig bis hundert Morgen unter sich haben. Einige Pächter erboten sich sogar, nachdem sie sehr reich geworden waren, gegen ihre Prinzipale zwei, drei, ja bis zu zehn Pachtungen gegen Vorschuß zu übernehmen. Die Gutsherren, verführt durch das Reizende dieser Anbietungen, ruinierten manche gute Familie, die durch die gierige Habsucht dieser Herren von Bauern zu Pächtern herabgewürdigt wurden. Alle Länderbesitzer folgen jetzt diesem bösen Beispiel, und folglich wird das Land mehr und mehr entvölkert, weil die verarmten Pächter teils nach Amerika gehen, teils als Manufakturisten in Städten, wo Fabriken und Manufakturen blühen, sich niederlassen." (J.R. Forster), Gemählde von England vom Jahre 1780, fortgesetzt von dem Herausgeber bis zum Jahre 1783. Aus dem Französischen übersetzt, 1784, 172. Vgl. zum neuesten Stand der Einhegungsforschung neben der unter Fußnote 20 angegebenen Literatur: W.E. Tate, The English Village Community and the Enclosure Movement, 1967; Thompson, English Working Class, $237 \mathrm{ff}$.; Lord Ernle (Prothero), English Farming Past and Present (1912). With new introductions by G. E. Fussel and O. R. McGregor, ${ }^{6} 1961$; V. M. Lavrovsky, Expropriation of English Peasantry in the 18th Century, EHR, 2nd ser. 9 1956-1957; E. Davies, The Small Landowners, 1780-1832, in the Light of Land Tax Assessments. EHR 1, 1927-1928, 87-113; D. D. Chambers, Enclosure and the Small Landowners, EHR 10, 1939 bis 1940, 118-137; ders., Enclosure and Labour Supply in the Industria Revolution. EHR, 2nd ser. 5, 1952-1953; J. Saville, Rural Depopulation in England and Wales, 1957.

22 F. W. Schubert, Handbuch der allgemeinen Staatskunde von Europa, 1/2: Das britische Weltreich, 1836.

${ }_{23}$ Nach englischen Forschungen ergibt sich folgende Steigerung der Armensteuern: $1780=$ zwei Millionen Pfund Sterling, $1803=$ vier Millionen Pfund Sterling, $1812=$ sechs Millionen Pfund Sterling. Vgl. Thompson, English Working Class, 245. sischen Krieges ist die Verelendung der Kleinpächter und Landarbeiter offen in Deutschland kritisiert worden ${ }^{24}$. Man war sich völlig einig darin, daß sich diese Gruppe in einer tiefen ölsonomischen wie sozialen Krisensituation befand. Es wurde zwar anerkannt, daß sich durch die "Enclosures" die agrarische Produktivität ungemein verbesserte und die traditionelle Immobilität des Landbewohners aufgehoben wurde, wodurch er dem Gesetz von Angebot und Nachfrage folgend auch höheren Löhnen und besseren Arbeitsbedingungen sich zuwenden konnte. Man lobte einerseits die physiokratischen Ideen eines Arthur Young und anderer englischer Agrarpioniere, bemerkte aber auch, daß die Aufteilung der Gemeinheiten und die Zusammenlegung zu größeren landwirtschaftlichen Betriebseinheiten den Zustand der "dürftigen Klasse" auf dem Lande verschlimmert habe ${ }^{25}$. So gibt es keinen Zweifel, daß deutschen Zeitgenossen schon vor dem Beginn der deutschen Agrarreformen die unaufhaltsame Erosion der alten englischen Agrarverfassung aufgefallen ist. Sie sahen, daß Dorf um Dorf gerade die Ärmsten ihre Existenzmöglichkeiten verloren und für den Verlust alter Rechte nicht entschädigt wurden. Schottische und walisische Bergbauern erschienen manchem, wie etwa Johanna Schopenhauer und Theodor von Schön, als Beispiele besonders extremer Pauperisierung ${ }^{26}$.

24 (Küttner), Beyträge 2, 89-94; Goede, England 5, 65, 317 und 375; Wendeborn, Reise durch einige Provinzen 1, 109, und 2, 23-29; (Hüttner u. a.), Artikel "London" in der Zeitschrift "London und Paris" 1-24, (1798-1810).

25 (Küttner), Beyträge 2, 90 ff.; Goede, England 5, 65; Wendeborn, Reise 1, 109; (Hüttner), Artikel "London“, in: Englische Miscellen 4, 150-151, und 25, 78-79. Vgl. dazu A. Thaer, Einleitung zur Kenntniß der englischen Landwirthschaft und ihrer neueren practischen und theoretischen Fortschritte in Rücksicht auf Vervollkommnung deutscher Landwirthschaft für denkende Landwirthe und Cameralisten, 3 Bde. und 1. Reg.-Bd., 1798-1804, und Engels, Lage der arbeitenden Klasse, $328 \mathrm{ff}$. (Kap. Das Ackerbauproletariat); W. Cobbet, Rural Rides, ed. E. W. Martin, 1958.

26 J. Schopenhauer, Reise durch England und Schottland, in: Sämtliche Schriften 15, 1830, 287-288; T. von Schön, Studienreisen eines jungen Staatsmannes in England am Schluß des vorigen Jahrhunderts, 1891, 83. Weitere Berichte über das Elend der schottischen und walisischen Landbevölkerung bringen: G. Holzenthal, Briefe über Deutschland, Frankreich, Spanien, die balearischen Inseln, das südliche Schottland und Holland. 
Die deutschen Eindrücke lassen sich aber weder für größere Regionen, noch für längere Zeitabschnitte verallgemeinern. Der schottische Farmer aus den öden Highlands (der sein Schicksal in seiner Erdhütte apathisch-zufrieden trägt) kann kaum mit dem anspruchsvollen „Ploughman“ oder dem im Akkord arbeitenden Ernteschnitter in der reichen Grafschaft Norfolk, geschweige denn mit einem irischen Wanderarbeiter in einen Topf geworfen werden. $\mathrm{Zu}$ viele antinomische Faktoren soziokultureller und materieiler Art verbieten es, die Gestalt eines durchschnittlichen Landarbeiters zu konstruieren. Das einzige, was sich mit Sicherheit sagen läßt, ist dies: Die Klagen über die agrarische Armut bedeuten zu verschiedenen Zeiten und zu verschiedenen Orten offenbar etwas Verschiedenes. Auch die Zugehörigkeit zu einer bestimmten Gruppe besagt wenig über den realen Lebensstandard. So zählen zu den Tagelöhnern (Day labourers, Journeymen) einerseits Frauen und Kinder mit Hungerlöhnen, andererseits Akkordarbeiter, die mit Spitzenlöhnen in der Erntezeit unter Umständen aus der Stadt geholt werden müssen ${ }^{27}$. Ständige Arbeitskräfte hatten zwar höhere Sicherheit des Arbeitsplatzes, aber auch die geringste Unabhängigkeit, die längsten Arbeitszeiten und die niedrigsten Barlöhne.

Eine erste zusammenfassende und nicht mehr an Einzeleindrücken orientierte Übersicht über die Situation des Landarbeiters ist erstmals 1840 in Deutschland gegeben worden, als übersetzte Auszüge aus einer Parlamentsenquête publiziert wurden'28. Diese

Geschrieben in den Jahren 1809 bis 1814; 1817, 209; Goede, England, Wales, Irland und Schottland 5, 317 und 337; J. G. Kohl, Reisen in Schottland 1, 1844, 134 f.; C. G. Carus, England und Schottland im Jahre 1844 2, 1845, 94, 101 und 255-256. Die älteste deutsche Beschreibung des schottischen Landarbeiters und Kleinpächters stammt von J. J. Volkmann, Neueste Reisen durch Schottland und Irland vorzüglich in Absicht auf die Naturgeschichte, Oekonomie, Manufacturen und Landsitze der Großen, 1784. Volkmann ist aber wenig originell, da er sich meistens auf Daniel Defoes Reiseberichte vom Anfang des 18. Jahrhunderts stützt.

27 Holzenthal, Briefe, 209; (Küttner), Beyträge 2, 90. Wendeborn, Reise 2, 19.

${ }^{28}$ Vom Ackerbaue und von dem Zustande der den Ackerbau treibenden Klassen in Irland und Großbritannien. Auszüge aus den amtlichen Untersuchungen und Akten, welche das Parlament vom Jahre 1833 bis bestätigte die generelle Verschlechterung der Lebenshaltung seit 1790 trotz gestiegener Löhne, die fast überall nur noch in Geld gegeben wurden. In ganz Südengland backte keine Landarbeiterfamilie mehr ihr Brot selbst. (Zum Vergleich: Um 1860 taten dies in den norddeutschen Zollvereinsstaaten noch zwei Drittel der Bevölkerung ${ }^{29}$.) Die Untersuchung zeigte auch, wie sich die Landarbeiter gegen die Auflösung der alten Agrarverfassung wehrten: Junge unverheiratete Burschen wanderten besonders nach 1820 aus, verdingten sich beim Kanal- oder Eisenbahnbau oder zogen in die Stadt. Die Masse der Allteren und Verheirateten blieb aber auf dem Land, fürchtete um ihre Zwergwirtschaft und ihr bißchen Besitz, fühlte sich trotz der Armut der traditionellen Lebensweise weiter verbunden. Auch scheute man wohl, auf dem städtischen Arbeitsmarkt mit dem verachteten irischen Immigranten zu konkurrieren, der sich überall als lohndrückend zeigte. Die heftigen Landarbeiterrevolten seit 1816 haben offenbar wenig an den Zuständen geändert. Der sie unmittelbar miterlebende Victor Aimé Huber vertrat dabei die überraschende These, der Fabrikarbeiter sei nicht der Teil des Proletariats, „welcher am vollständigsten und feindseligsten außerhalb der Grenzen der gesetzlichen und sozialen Zivilisation steht" - das ländliche Proletariat sei viel negativer, brutaler, zerstörender und zeige viel weniger Eingewöhnung in die neue Ordnung ${ }^{30}$. Die Brandstiftungen und Zerstörungen der Dreschmaschinen wurden von ihm mit dem Hinweis verteidigt, dies stelle im Befrußtsein des Landarbeiters nicht mehr als ein Wildfrevel oder Holzdiebstahl dar, nämlich die Inanspruchnahme eines vorenthaltenen Rechts. Die Forderungen drehten sich auch um Löhne und niedrigere Lebensmittelpreise, meistens aber doch nur um Landbesitz. Friedrich Engels, mit dessen

auf den heutigen Tag öffentlich bekannt gemacht hat. Sammt einigen Bemerkungen der Herausgeber, 2 Bde., 1840; soweit man erkennen kann, ist Engels dieser wichtige Parlamentsbericht unbekannt geblieben.

${ }_{29} \mathrm{G}$. von Viebahn, Statistik des zollvereinten und nördlichen Deutschlands $2,1862,860$.

30 V.A. Huber, Erinnerungen, Eindrücke und Betrachtungen eines Reisenden, Janus 2, 1845, 651; vgl. M. Dutt, 'The Agricultural Labourer's Revolt of 1830 in Kent, Surry and Sussex, Phil. Diss. London 1967; A. J. Peacock, Bread or Blood. The Agrarian Riots in East Anglia 1816, 1965; Hasbach, Geschichte des englischen Landarbeiters, 192 ff.; Thompson, English Working Class, 241. 
Schilderungen vom englischen "Ackerbauproletariat" so sachverständige Kenner der Landwirtschaft wie Alexander von Lengerke und Hermann Johann Friedrich Schulze-Gaevernitz übereinstimmten, wurde nicht müde, den englischen Landarbeiter mit dem Fabrikarbeiter faktisch gleichzustellen, da auch er nur einen Taglohn erhält und davon allein leben muß31. In den Landarbeiterrevolten erblickte er den Beginn eines "sozialen Krieges“, der $\mathrm{zu}$ einer Vereinigung mit den Industrieproletariern führen würde. Dieser Ansicht konnten sich die anderen Zeitgenossen freilich nicht anschließen. Bezeichnenderweise sah Engels in der starken Religiosität auf dem Lande selbst ein großes Hindernis für eine solche Entwicklung. Der Landarbeiter bildete ohne Zweifel zahlenmäßig die größte Gruppe unter den sozialen Unterschichten in der britischen Frühindustrialisierung. Zunehmende Ubervölkerung und abnehmende Beschäftigungsmöglichkeiten in der ländlichen Hausindustrie sowie Verlust der Eigenversorgung hatten trotz verbesserter Armenfürsorge ihm eine absolut höhere Massenarmut beschert, die sich allerdings regional, temporal und sozial unterschiedlich auswirkte. Offenbar reagierte die englische Landwirtschaft sehr unelastisch auf die veränderte Nachfrage nach Arbeitskräften. Die bekannte These, die Einhegungen und Gemeinheitsteilungen hätten die ländlichen Gegenden depopulationiert und direkt zur Bildung der proletarischen Fabrikarbeitermassen in den Städten geführt, findet in der zeitgenössischen deutschen Englandliteratur wie auch in den neuesten englischen Forschungen wenig Stützen ${ }^{32}$. Es scheint, als wenn hier vieles zu einfach gesehen worden ist. Zwischen den Einhegungen und der sozialen Umschichtung von der Landwirtschaft in die Industrie lassen sich statistisch kaum Korrelationen geben. Soweit man er-

31 Engels, Lage der arbeitenden Klasse, 328-342; H. J. F. Schulze(Gaevernitz), Nationalöconomische Bilder aus Englands Volksleben. Mit besonderer Berücksichtigung der landwirthschaftlichen und industriellen Verhältnisse, 1853, 155-163; A. von Lengerke, Landwirthschaftliche Jahresschrift. Eine geographisch-statistisch-volkswirthschaftliche Umschau auf dem Gebiet des Landbaues außerhalb Deutschlands, 1852; ders., Art. England in landwirthschaftlicher Beziehung, in: A. von Lengerke (Hrsg.), Landwirthschaftliches Conversations-Lexicon für Praktiker und Laien 1, 1837, 762-798.

32 J. D. Chambers, Enclosure and Labour Supply in the Industrial Revolution, EHR, 2nd ser. 5, 1952-1953. kennen kann, setzte die große Landflucht erst nach 1850 ein. Agrarische Übervölkerung und Ausbau der Industrie haben anscheinend zunächst relativ beziehungslos nebeneinander gestanden.

Sieht man von der statistisch schlecht faßbaren städtischen und ländlichen Armut sowie den Dienstboten $a b$, dann sind neben den Landarbeitern die Handwerker und Heimarbeiter zwischen 1780 und 1840 quantitativ die größte Gruppe gewesen ${ }^{33}$. Es kann keinen Zweifel geben, daß bis zur Mitte des 19. Jahrhunderts die Masse der gewerblichen Arbeitskräfte auch in England nicht in zentralisierten Produktionswerkstätten, sondern zu Hause oder in benachbarten Werkräumen mit kleingewerblichem Charakter gearbeitet hat. Daneben betätigte man sich im Bergbau- und Hüttenwesen, auf dem Bau oder in den Docks, aber auch hier hatte sich infolge fehlender Mechanisierung bis auf Ausnahmen wenig an den traditionellen Arbeitsvollzügen geändert ${ }^{34}$. Die deutschen Beobachter hatten zwar bemerkt, daß Menschen in die neuen Fabriken strömten, konnten aber die Verschiebung in den Berufsstrukturen der "arbeitenden Klassen“ zunächst in den wahren Proportionen nicht erfassen. Auch Friedrich Engels ist das noch nicht gelungen. Dies wirkt verzeihlicher, wenn man weiß, daß auch die englische Statistik darüber lange im unklaren war. Eine besondere Schwierigkeit lag darin, daß man in der Sprache der Zeit die Begriffe noch nicht sauber trennte. So sprach man häufig kon „Fabrikarbeitern", obwohl in Wahrheit Beschäftigte in einer Manufaktur, im Verlag oder im Handwerk gemeint waren. Umgekehrt behielten alte Handwerksbezeichnungen in der Fabrik noch ihre Geltung, obwohl diese Beschäftigten zu unselbständigen Lohnarbeitern degradiert worden waren. Auch die Nachzeichnung des Bildes bei den Handwerkern und Heimarbeitern ist schwierig, weil sich hinter dem Berufsschild „Artisan", „Outworker", „Weaver", „Miner" etc. sozial wie ökonomisch etwas sehr Verschiedenes verbergen konnte. Die Skala reicht von dem wohlsituierten Handwerksmeister als Kleinunternehmer (z. B. Master Weaver) und dem wegen seiner Spezialkenntnisse hochbezahlten Mechaniker bis zu

33 Thompson, English Working Class, 344.

34 W. M. Sterne, The Porters of London, 1960; T. S. Ashton, The Coal Miners of the 18th Century, Economic Journal (Suppl.) 1, 1928, 325; R. Postgate, The Builder's History, 1923. 
dem armen dörflichen Nagelschmied und den von einem Zwischenmeister erbarmungslos ausgebeuteten Schneidergesellen. In der Statistik sind alle diese Gruppen nicht sauber getrennt.

Das Handwerk hatte in London seinen Hauptsitz. Neben den „ehrlichen Zünften" (honourable trades) wie den Juwelieren, Uhrmachern, Druckern, Bäckern, Schneidern usw. standen die „unehrlichen" wie etwa die Bierbrauer und Seidenweber. Letztere müssen nach deutschen Zeugnissen schon in der Mitte des 18. Jahrhunderts als proletaroid angesehen werden, um einen vorzüglichen Sombartschen Begriff aufzugreifen. Durch die Einführung der wasserangetriebenen Seidenspinn- und Webmaschinen seit 1718, die durch James Brindley in den fünfziger Jahren wesentlich verbessert wurden, ereignete sich hier die erste technische Revolution im Textilgewerbe. Verlust des handwerklichen Status, Ausbreitung kapitalintensiver Verlags- und Manufaktursysteme und Uberangebot billiger Arbeitskraft infolge Arbeitslosigkeit und Lohndrückerei sind hier zuerst $\mathrm{zu}$ beobachten gewesen. Lange vor Friedrich Engels hat Vater Leopold Mozart in Briefen an seinen Salzburger Hauswirt 1763 die „Brotkrawalle“ der etwa 85.000 Seidenweber in dem Londoner Stadtteil Spitalsfield geschildert, die mit Waffengewalt niedergeschlagen werden mußten ${ }^{35}$. Noch um 1850 waren alle Versuche gescheitert, die durch Verwandtschaft, Schulden und Lebensgewohnheiten eng verketteten, armen Weberfamilien umzusiedeln oder in anderen Berufen unterzubringen ${ }^{36}$.

Ein ganz anderes Bild ergibt sich dagegen bei der Betrachtung der Kleineisenindustrie in Birmingham. Einer der besten deutschen Englandkenner um 1800 meint, es gebe wohl keine Stadt in ganz Großbritannien, wo sich die "niedrige Klasse" wohler befinde als gerade hier ${ }^{37}$. Infolge der großen Arbeitsteilung ist wie in Sheffield die kleine Werkstatt vorherrschend geblieben. Der Unter-

35 W. A. Mozart, Briefe und Aufzeichnungen. Hrsg. von der Internationalen Stiftung Mozarteum Salzburg. Gesammelt und erläutert von W. A. Bauer und O. E. Deutsch 1, 1962, 186.

36 M. Schlesinger, Wanderungen durch London 2, 1853, 223-226; vgl. Engels, Lage der arbeitenden Klasse, 76, 96 und 265; G. B. Hertz, The English Silk Industry in the 18th Century, English Historical Review 1909; W. H. Chaloner, People and Industries, 1963, 8-20.

37 Goede, England 5, 256; S. Timmings (ed.), Birmingham and the Midland Hardware District, 1866; G. Stuart, The Wheelwright's Shop, 1923. schied zwischen dem Kleinmeister und dem gelernten Handwerker ist gering. Viele besitzen ein Grundstück und können sich selbständig machen. Man lebt noch ganz in alten Sitten, feiert gemeinsame Feste und treibt großen Aufwand in Essen und Trinken, der Lebenszuschnitt bleibt aber bescheiden. Die alten Handwerksgeheimnisse werden in strenger Abschließung nach draußen nur an die eigenen Zunftgenossen weitergegeben. Keine Schulen oder technischen Akademien können dieses Wissensmonopol streitig machen. Zunftartiges Kastendenken, vermischt mit Anspruch auf hohes Sozialprestige, geht fast nahtlos in die entstehenden "Trade Unions" über. Die Gesellschaft wird nicht, wie die Webbs später beklagten, hier in Unternehmer und Arbeiter horizontal aufgespalten, sondern bleibt vertikal nach Gewerken (Trades) geschieden, die egoistisch über ihre Privilegien wachen. Auch in Birmingham gibt es „Pöbel“, arme Nagelschmiede und die große Maschinenbauanstalt von Watt \& Boulton im Vorort Soho, aber vorherrschend ist das Bild insgesamt geglückter Assimilation des Handwerks an die Industrialisierung ${ }^{38}$.

In der bisherigen Literatur sind aber nun nicht die Landarbeiter und Handwerker, sondern der Heimarbeiter und dort ganz besonders der Handweber als die eigentlich tragische Figur der Frühindustrialisierung bezeichnet worden. Edward P. Thompson hat neuerdings vier Typen des Handwebers im ausgehenden 18. Jahrhundert unterschieden: erstens den auf Kundenbestellung arbeitenden unabhängigen Handwerksmeister, zweitens den für mehrere Meister tätigen quasi-autonomen Handweber, drittens den von einem einzigen Meister abhängigen Tagelöhner, der zu Hause an seinem Webstuhl oder in einer fremden Werkstatt sitzt, und schließlich viertens den zeitweise in Nebenerwerb tätigen Landarbeiter. In der historischen Realität laufen die Arten durcheinander, doch geht die Entwicklung tendenziell zum dritten Typ, der als „dishonourable" gilt ${ }^{39}$. Erstaunlich frühzeitig haben deutsche Beobachter, wie der Pädagoge Johann Heinrich Campe schon 1802, das Thema des "überhandnehmenden Maschinenwesens" bei den

${ }^{38}$ Reise durch England und Schottland, in: Beiträge zur Völker- und Länderkunde. Hrsg. von (J. R. Forster) und M. Sprengel, Theil 14, 1790, 178-184; T.K. Derry, Repeal of the Apprenticeship Clauses of the Statute of Apprentices. EHR 3, 1931-1932.

${ }^{30}$ Thompson, English Working Class, 297. 
"Handloom weavers" immer wieder variiert ${ }^{40}$. Stellte die eine Seite den Nutzen, den das „bewundernswürdige Kunstgetriebe der Maschinen" brachte, in den Vordergrund, so betrachtete die andere dies als Entheiligung aller Verhältnisse von Menschenwohl

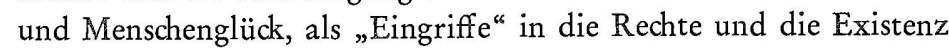
der arbeitenden Klasse, als „Verdienstraub der Reichen " und die Maschinenstürmerei als legitime Notwehr ${ }^{41}$. Eine nicht mehr an Zufallseindrücken, sondern an empirisch-statistischen Grundlagen orientierte Übersicht ist aber erst zwischen 1835 und 1845 deutschen Englandschriftstellern gelungen ${ }^{42}$. Carl Theodòr Kleinschrod, Oberberg- und Salinenrat, zeitweise Inspektor der Nymphenburger Porzellanmanufaktur, dann Referent für Landwirtschaft, Handel und Gewerbe sowie Statistik im bayerischen Innenministerium, hat die Tragödie des englischen Handwebers wohl am ersten und gedanklich am tiefsten durchdrungen. Auf Grund seiner vier "Gewerbsreisen“ ins westliche Ausland erkannte er schon 1834/35 zwei Entwicklungsphasen bei der Entstehung der neuen industriellen Lohnarbeiterschaft: In der ersten Phase erfolgte die Umwandlung des selbständigen hausgewerblichen Handspinners mit landwirtschaftlicher Selbstversorgung sowie der verdrängten Klein-

${ }^{40} \mathrm{~J} . \mathrm{H}$. Campe, Reise durch Engldnd und Frankreich in Briefen an einen jungen Freund 2, 1803, 11-17.

${ }_{41}$ (R. Greger?), Englands Industrie und die mechanischen Erfindungen sind das Verderben des festen Landes. Dargestellt zur Beherzigung für die Mächtigen und Reichen wegen der verdienstlosen Armen, 1817, 93-96; Konrad Engelbert Oelsner an Varnhagen von Ense am 6.9.1816, in: L. Assing (Hrsg.), Briefwechsel zwischen Varnhagen von Ense und Oelsner nebst Briefen an Rahel 1, 1865, 17.

$42 \mathrm{C}$. Th. Kleinschrod, Großbritanniens Gesetzgebung in Gewerbe, Handel und innere Communicationsmittel statistisch und staatswirthschaftlich erläutert, 1836; ders., Der Pauperism in England in legislativen, administrativen und statistischen Beziehungen, 1845; ders., Die neue Armengesetzgebung Englands und Irlands in ihrem zehnjährigen Vollzuge, 1849; ders., Der Pauperism in England, 2. Fortsetzung, 1853; F. von Raumer, England im Jahre 1835 2, 1836, 10 ff.; T. F. Kniewel, Reiseskizzen, vornehmlich aus dem Heerlager der Kirche, gesammelt auf einer Reise in England, Frankreich, Belgien, Schweiz, Oberitalien, Deutschland, im Jahre 1842 1, 1843, $342 \mathrm{ff}$.; Engels, Lage der arbeitenden Klasse, 202 ff., 256 ff.; K. H. Rau, Besprechung der Schrift: Handloom Weavers, Report of the Commissioners. Presented to both Houses of Parliament by Command of Her Majesty, London 1841, in: Archiv der politischen Oekonomie und Polizeywissenschaft, N. F. 1, 1843, 275 ff. pächter und Freeholder in abhängige Lohnarbeiter. In einer Engpaßphase des Garnmangels waren diese Gruppen durch hohe Lohnspannen zur Veräußerung ihres Landbesitzes und zum Kauf der unkomplizierten und relativ billigen Spinnmaschinen verleitet worden. Mit zunehmender Mechanisierung sanken dann die Produktpreise, während gleichzeitig durch den technischen Fortschritt Neuinvestitionen bei den Maschinen immer teurer wurden. In dieser Scherensituation von steigenden Investitionspreisen und sinkenden Erlösen für die Produkte konnten nur die Kapitalkräftigsten und Tüchtigsten ihre Selbständigkeit wahren, während die anderen zur "neuen Classe" der Lohnarbeiter herabsanken ${ }^{43}$. Ein analoger Prozeß ist dann nach Kleinschrod bei der Weberei zu beobachten gewesen. Auch hier kann man nicht einfach sagen, der Weber sei ein Opfer der Maschinisierung und des Fabrikensystems geworden: Ganz im Gegenteil stieg zunächst die Zahl der Handweber in der Baumwoll- und Wollindustrie zwischen 1780 und 1830 durch die Einführung der Spinnmaschinen kräftig an, da das viele billige Garn nach vielen zusätzlichen Weberhänden suchte ${ }^{44}$. Der Untergang des Handspinners bedeutete nach Kleinschrod zunächst den Aufstieg des Handwebers. Erst dann machten die Verbesserungen der mechanischen Weberei die Handweber ebenfalls überflüssig und erzeugten damit auf dem Arbeitsmarkt eine wiederum neue Strukturkrise. Ahnliche Vorgänge wiederholten sich parallel, meistens aber mit temporärer Phasenverschiebung in der Woll- und Leinenindustrie. Auch hier blähten Maschinen jedesmal diese zunächst auf, um die Handarbeit dann um so vernichtender zu treffen ${ }^{45}$.

43 Kleinschrod, Gesetzgebung, $167 \mathrm{ff}$.

44 Ebd., 187.

45 Über die Entwicklung der Arbeitsverhältnisse in der britischen Textilindustrie zwischen 1750 und 1850 gibt es bekanntlich ein großes Schrifttum. Als besonders wichtig ist unter anderem $\mathrm{zu}$ nennen: E. Baines, Account of the Woollen Manufacture of England (Reprint), 1970; H. Forbes, Rise, Progress and Present State of the Worsted Manufactures, 1852; J. James, History of the Worsted Manufactures, 1857; J. Burney, History of Wool and Woolcombing, 1889; D. M. Hunter, The West of England's Wool Industry, 1910; H. Heaton, Yorkshire Woolen and Worsted Industries, 1920; ders., Benjamin Gott and the Industrial Revolution in Yorkshire, EHR 3, 1931-1932, 45-66; W. G. Hopkins, Industry, Trade and People in Exeter, 1688-1800, with special Reference of the Serge Industry, 1935; M. Carus-Wilson, The

17 Kellenbenz, Wirtschaftspolitik 
Diese Prozesse der Mechanisierung und Zentralisierung sind wie immer nach Zeit, Region und Gewerbezweig stark zu differenzieren. So war in der Baumwollweberei die alte Zunftverfassung schon in der Mitte des 18. Jahrhunderts durch die Aufhebung der Lehrlingsbeschränkungen praktisch liberalisiert worden. Auf dem Höhepunkt der Einhegungswellen nach 1790 stürzten sich landlos gewordene Kleinpächter und Landarbeiter, irische Einwanderer, entlassene Soldaten, Armenhäusler und vor allem Frauen und Kinder auf das einfach zu erlernende, monotone Baumwollweben. Die Handweberei war deshalb so attraktiv, weil man zu Hause arbeiten und seine Zeit beliebig einteilen konnte. Ein zwölfjähriger Knabe konnte bereits nach acht Wochen perfekt weben; nur das Aufziehen der Ketten erforderte einige Handfertigkeit. Der Verleger lieferte das Garn und nahm gegen einen Stücklohn die gewebten Stücke ab. Das Fehlen einer Lehrzeit und die Chance einer frühen Heirat waren ebenfalls anziehend. Kinder wirkten sich als Hilfskräfte nur günstig aus. Dies ist in Wahrheit die "goldene

English Cloth Industry, EHR 14, 1944, 32-50; E. Lipson, A Short History of Wool and its Manufacture, mainly in England, 1953; K. G. Pointing, A History of the West of England Cloth Industry, 1957; J. de L.Man, Clothiers and Weavers in Wiltshire during the 18th Century, in: L. S. Pressnell (Hrsg.), Studies in the Industrial Revolution, presented to T.S. Ashton, 1960; R. G. Pelham, Fulling Mills: A Study to the Application of Water Power to the Woolen Industry, 1958; J. Prest, The Industrial Revolution in Coventry, 1960; P. B. Sidney, The Growth of Textile Factories in Derbyshire during the 18th Century, 1965; A. Ure, The Philosophy of Manufacture: or Exposition of the Scientific, Moral and Commercial Economy of the Factory System of Great Britain, 1835 (Deutsch: Das Fabrikwesen in wissenschaftlicher, moralischer und kommerzieller Hinsicht, 1835); ders., The Cotton Manufacture of Great Britain, 1836; E. Baines, History of the Cotton Manufacture in Great Britain... (1835), Reprint with a biographical Introduction by W. H. Chaloner, 21966 ; J. Wade, History of the Middle and Working Class, ${ }^{3} 1835$; J. Fielden, The Curse of the Factory System, 1836; W. Cudworth, Conditions of the Industrial Classes of Bradford and District, 1887; W. Scruton, Bradford Fifty Years Ago, 1897; S. J. Chapman, The Lancashire Cotton Industry, 1904; J. L. Hammond and B. Hammond, The Town Labourer, 1760-1832, 2 vols. (Reprint), 1949; A. P. Wadsworth and J.L. de Man, The Cotton Trade and Industrial Lancashire, 1931; N. J. Smelser, Social Change of Industrial Revolution. An Application of the Theory to the Lancashire Cotton Industry, 1770-1840, 1959.
Zeit" des Handwebers gewesen, von der der Arzt Peter Gaskell in seinem Buch über die englische Fabrikbevölkerung 1835 gesprochen hat und das dann Friedrich Engels in so reichem Maße als Vorbild diente ${ }^{46}$. Aber dieses "goldene Zeitalter" ist, wie die englische Forschung längst herausgefunden hat, nur ein Mythos gewesen ${ }^{47}$. Engels' historische Kenntnisse sind an diesem Punkt ausgesprochen defekt. Zwar war das Einkommen des Handwebers vor 1800 relativ höher als 1830, der alte handwerkliche Status durch das Einströmen fremder Arbeitskräfte aber längst verlorengegangen.

Die Not des Handwebers, der bald in mancher Fabrik den „eigentlichen Kern der gemeinen Arbeiterklassen“ bildete, ist in deutschen Reiseberichten so geschildert worden, daß Engels' düsteres Gemälde manchmal noch bei weitem übertroffen wird ${ }^{48}$. Ein Blick in die Statistiken von Kleinschrod und eines von William Nassau Senior verfaßten sowie von dem deutschen Ökonomen Karl Heinrich Rau kritisch rezensierten Parlamentsberichts zeigt freilich, daß die Dampfkraft eigentlich erstaunlich langsam vorgedrungen ist. Die technische Erfindung (Inovation), ihre ökonomische Durchsetzung (Innovation) und schließlich ihre massenhafte Anwendung (Imitation) klaffen zeitlich weit auseinander49. So gab es 1813 in Großbritannien 2400 Maschinenwebstühle, 1820

46 P. Gaskell, The Manufacturing Population of England, its moral, social and physical Conditions, and the Change which has arisen from the Use of Steam Machinery; with an Examination of Infant Labour, 1833; vgl. dazu W. O. Henderson and W.H. Chaloner, Friedrich Engels in Manchester, Memoirs and Proceedings of the Manchester Literary and Philosophical Society 48, 1956-1957, 13-29; M. Jenkins, Frederick Engels in Manchester, 1951; W.O. Henderson and W.H. Chaloner, Engels and the England of the 1840's, History Today 6, 1956; G. Carlton, Friedrich Engels. The Shadow Prophet, 1965; W. O. Henderson and W. H. Chaloner, Friedrich Engels and the England of the "Hungry Forties", in: Arthur Seldon (Hrsg.), The Long Debate on Poverty, 1972.

${ }_{47}$ D. George, England in Transition, 1953, 63.

48 Vgl. z. B. die anschauliche Schilderung des deutschen Pastors Kniewel, Reiseskizzen, $342 \mathrm{ff}$. Der Autor stützt sich dabei gelegentlich auf das Buch von C. Taylor, Voyage in Lancashire, 1842.

49 G. Mensch, Zur Dynamik des technischen Fortschritts, Zeitschrift für Betriebswirtschaft 41, 1971, 295-314; J. Schmookler, Invention and Economic Growth, 1966; J. Sewkes, D. Sawers and R. Stillerman, The Sources of Invention, 1960. 
erst 14.000. Nach 1825 erfolgte dann eine starke Zunahme, so daß 1829 schon 45.000 und 183510.000 Power Looms in der Baumwollindustrie gezählt wurden, in der Jahrhundertmitte war dann eine Viertelmillion überschritten. Dagegen gab es aber 1835 allein in der Baumwollindustrie noch 250.000 Handweber, 840.000 in der gesamten britischen Textilindustrie ${ }^{50}$. Auf Grund dieser Zahlen läßt sich nur vermuten, daß die „Dampfstühle“ bis $1825 \mathrm{mehr}$ ein massenpsychologisches als ein ökonomisches Problem gewesen sind. Man kann sogar die These daran knüpfen, daß der künstlich erzeugte Überfluß an billigen Arbeitskräften in der Textilindustrie die Mechanisierung im ganzen hier verzögert hat, da die Unternehmer verlockt wurden, ihre Maschineninvestitionen hinauszuschieben. Möglicherweise hätte eine raschere Industrialisierung bei gleichen Einflußfaktoren besonders das Elend des handarbeitenden Heimwebers abgekürzt ${ }^{51}$. Senior hebt in seinem Bericht hervor, daß manche Zweige der Textilindustrie auch ganz spät in den Sog der Industrialisierung gerieten. So blieb das Weben feiner gemusterter Zeuge und Teppiche bis über 1850 hinaus reine Handarbeit. Der Jacquardwebstuhl war zunächst nicht zu ersetzen. Wie im anderen Handwerk konnten sich auch hier gewisse alte Eliten halten ${ }^{52}$.

Die knappe Abhandlung verbietet es, noch andere in der nichtmechanisierten Produktion stehende Berufsgruppen, wie etwa die Berg- und Hüttenarbeiter, die Töpfer in Staffordshire oder das Baugewerbe bzw. einzelne Dienstleistungsberufe (Fuhrleute, Fluß-

50 Meidinger, Das Britische Reich, 344; Kleinschrod, Gewerbegesetzgebung, 188. (Die Zahlen wurden aus der berühmten Statistik von G. R. Porter, The Progress of the Nation, ${ }^{\mathbf{3} 1851}$, 411, übernommen.)

51 Ashton schreibt zu diesem Punkt: „It is sometimes suggested that the evils of the industrial revolution were due to the rapidity with which it proceeded: the case of the domestic textile workers suggest the exact opposite. If there had been in weaving a man of the type of Arkwright, if rates of interest had remained low, if there had been no immigration and no Poor Law allowance, the transfer to the factory have been effected quickly and with less suffering. As it was, large numbers of hand workers continued for more than a generation to fight a loosing battle against the power of steam." Vgl. T.S. Ashton, The Industrial Revolution, 1760-1830, 1948, 117.

52 Rau, Handloom-Weavers, 275. und Kanalschiffer, Seeleute) zu betrachten, die zum Teil einen hohen Lebensstandard wahren konnten ${ }^{53}$. Abschließend ist es nur noch möglich, einen Blick auf die Fabrikarbeit selbst mit besonderer Rücksicht auf die Kinderarbeit zu werfen. Letztere gehört zu den dunkelsten Kapiteln der britischen Frühindustrialisierung und ist sowohl von Engels und Marx als auch von anderen deutschen Englandschriftstellern in den Mittelpunkt der Betrachtungen über die aufkommende Fabrikindustrie gestellt worden ${ }^{54}$. Das Problem ist ungemein komplex: Die Kinderarbeit ist bekanntlich keine Erfindung der Fabrikindustrie gewesen, sondern reicht gerade in England weit zurück. Schon ein elisabethanisches Lehrlingsstatut von 1601 besagte, daß jedes Armenkind in eine Lehre gegeben werden solle. Im 18. Jahrhundert wuchs die Nachfrage nach Armenkindern, da der Lehrherr aus der Armenkasse des Kirchspiels eine Prämie bekam ${ }^{55}$. Die Ausnutzung der kindlichen Arbeitskraft stand schon vor der Mitte des 18. Jahrhunderts in

53 Bemerkenswerterweise beschäftigt sich Friedrich Engels überhaupt nicht mit diesen Berufsgruppen und umgeht insbesondere die ganze Schwerindustrie. In seinem Kapitel „Bergwerksproletariat" geht er lediglich auf das Problem der Kinderarbeit und die Gesundheits- und Moralitätsverhältnisse ein. Er muß zugeben, daß z. B. der erwachsene Bergarbeiter über einen vergleichsweise hohen Lebensstandard verfügte. Engels hat Bergbau und Hüttenwesen in England persönlich nie kennengelernt. Vgl. dagegen die anschaulichen deutschen Beschreibungen von J. G. Kohl, Reisen in England und Wales 2, $22 \mathrm{f}$., $43 \mathrm{f}$; Carus, England und Schottland 2, $48 \mathrm{f}$., 57; Meidinger, Reisen 1, 258. Vgl. ferner Engels, Lage der arbeitenden Klasse, 313; Th. S. Ashton, The Coal Miners of the 18th Century, Economic Journal (Suppl.) 1, 1928, 325; ders., Iron and Steel in the Industrial Revolution, 31963; R. H. Campbell, Carron Company, 1961; A. Birch, The Economic History of the Iron and Steel Industry 1784-1879, 1967; G. H. Hardach, Der soziale Status des Arbeiters in der Frühindustrialisierung. Eine Untersuchung über die Arbeitnehmer in der französischen eisenschaffenden Industrie zwischen 1800 und $1870,1969$.

54 Engels, Lage der arbeitenden Klasse, 209 ff.; K. Marx, Das Kapital. Hrsg, vom Marx-Engels-Lenin-Institut Moskau (Nachdruck der MEGAAusgabe von 1932) 3, 51956, 108 ff.; I. Pinchbeck - M. Hewitt, Children in English Society, 1969; I. Pinchbeck, Woman Workers in the Industrial Revolution, 1930; M. Hewitt, Wives and Mothers in the Victorian Industry, s. a. (1958); Thompson, English Working Class, 366-384.

$55 \mathrm{~J}$. Kulischer, Allgemeine Wirtschaftsgeschichte des Mittelalters und der Neuzeit 2, $21958,458$. 
manchen Berufszweigen nicht hinter den Baumwollspinnereien zurück, wobei an die Figur des kleinen Kaminkehrers und Schiffsjungen besonders zu denken ist. Die „optimistische Schule“ in der englischen Wirtschaftsgeschichte hat daher gegenüber der „pessimistischen Richtung" (die auf Carlyle, Engels, die Webbs und die Hammonds zurückgeht) geltend gemacht, die Kinderarbeit sei von den frühen Fakrikunternehmern gleichsam nur aus der vorindustriellen Wirtschaft ebenso wie die langen Arbeitszeiten tnd der patriarchalische Führungsstil übernommen worden, das Bewußtsein von der Inhumanität der Kinderarbeit erst später erwacht. Als aber die ersten Proteste und Parlamentsuntersuchungen kamen, fingen sich die Zustände schon zu bessern an. Was sagen nun die zeitgenössischen deutschen Englandberichte?

Die ersten deutschen Notizen über die Kinderarbeit in der mechanischen Spinnerei von Richard Arkwright in Cromford, die von einem mit englischen Verhältnissen gut vertrauten deutschen Erzieher stammen, loben bezeichnenderweise in völlig naiver Weise, daß hier ein vierjähriges (!) Kind die Arbeit eines erwachsenen Mannes tun könne ${ }^{56}$. Der Betrieb spare Lohn ein, und die Kinder würden statt zur Last zum Segen. Offenbar wurde hier noch jede den „Müßiggang “ einschränkende „Industrie" für nützlich gehalten. Erst ab etwa 1820 wird die Kinderarbeit in den deutschen Anglicana als ein Reformanliegen empfunden. Kleinschrod brachte zu dieser Zeit das Problem auf die Kurzformel, daß die Arbeiter einerseits auf den Verdienst ihrer Kinder, die Baumwollfabriken aber auf die billige Arbeitskraft angewiesen waren ${ }^{57}$. Die Kinderarbeit wurde zunächst dadurch befördert, daß die mechanischen Spinnereien mit Wasserantrieb in meist entlegenen Gebieten an den Ufern eines schnell fließenden Stromes gebaut wurden. In großen Herden wurden Hunderte von Armenkindern aus den "Work Houses" dorthin getrieben. Die ungewöhnlich niedrigen Arbeitsräume, die kleinen Maschinen, Schlaf- und Betsäle zeigen, daß man sich von Beginn an nur auf Kinderarbeit eingestellt hatte. Schon 1796 beschäftigte die Peelsche Baumwollspinnerei mehr als 1000 Kinder. „Ehrliche" Handwerker waren für solche erste frühindustrielle Fabriken, die wie die Manufak-

${ }^{56}$ (Küttner), Beyträge 1, $49 \mathrm{f}$.

57 Kleinschrod, Gewerbegesetzgebung, 180. turen im Geruch eines Arbeits- und Zuchthauses standen und auch so aussahen, schwer zu bekommen. Zudem hatte die Arbeit in den weit entlegenen Gebieten den Charakter einer Verbannung. Erwachsene Armenhäusler, irische Einwanderer, Tramps usw. konnten sich ebenso wie ein Handwerker oder Heimarbeiter schwer an den Gedanken einer Fabrikdisziplin gewöhnen, machten gerne ihren "blauen Montag" oder liefen einfach wieder davon, wenn sie das erste Geld bekommen hatten. Nur der hochqualifizierte Aufseher (Factor) und Mechaniker (Engineer) als die von der Konkurrenz odēr sogar vom Ausland umworbenen Fachkräfte mußten mit Häusern, hohen Löhnen und langzeitigen Verträgen angelockt werden. Kinder und solche Arbeitskräfte bildeten die Bewohner dieser ersten patriarchalischen "Fabrikdörfer", wofür Richard Owens New Lanark ein gutes Beispiel ist ${ }^{58}$. Erst mit der Umstellung auf Dampfkraft wurden Spinnereien in städtischen Regionen gebaut, wodurch relativ mehr männliche erwachsene Arbeiter Beschäftigung fanden. In Maschinenspinnereien blieben Frauen, Jugendliche und Kinder aber noch dominierend, weil bei den fallenden Preisen und der scharfen Konkurrenz der Lohnkostenanteil im Produkt für die Betriebe existenzentscheidend war.

Das Ansteigen der industriellen Kinderarbeit hängt aber auch mit der Bevölkerungsvermehrung zusammen, die sich bekanntlich $\mathrm{zu}$ einem großen Teil in einer geringeren Säuglings- und Kindersterblichkeit sowie in höherer Gebürtigkeit ausdrückte. Infolgedessen wuchs gerade in den ersten Dezennien der Industrialisierung der Anteil der Kinder und Jugendlichen in der Bevölkerungspyramide stark an. Als die mechanischen Baumwollspinnereien sich im großen Stil durchsetzten, gab es einen starken Überhang in den untersten Jahrgängen. Die Fabriken konnten nun erstmals Tausende von Armenkindern, bei denen Waisen und Halbwaisen eine große Rolle spielten, an Stelle der traditionellen Gelegenheitsbieschäftigung nun eine dauernde Beschäftigung geben, wenngleich

58 Die Räumlichkeiten der Owenschen Fabrik, die der Verfasser 1967 besichtigen konnte, lassen noch deutlich die Funktionen aus der Zeit der Frühindustrialisierung erkennen. Für die sachverständige Führung und Überlassung von wertvollem historischen Bildmaterial sei an dieser Stelle den Gourope Works Ltd. als der jetzigen Eigentümerin gedankt. Ein anderes solches "Fabrikdorf“ schildert R. S. Fitton - A. P. Wadsworth, The Strutts and the Arkwrights, 1758-1830, 1958. 
vom heutigen Standpunkt in einer wenig humanen Weise ${ }^{59}$. Die seit 1780 statistisch belegbare Kinderflut und die ökonomischtechnische Entwicklung speziell in der Baumwollindustrie sind historisch zufällig zusammengestoßen und bilden den harten Kern der Kinderarbeitsfrage. Die Sozialkritik des 19. Jahrhunderts hat diese Zusammenhänge noch nicht erkennen können und die Kinderund Frauenarbeit anfangs fälschlicherweise für ein Charakteristikum des "Maschinenzeitalters" überhaupt gehalten. Kleinschrod, der alle erreichbaren Materialien darüber studierte, hat die Nachteile der industriellen Kinderarbeit wie folgt gesehen: Die zwölfstündige Arbeitszeit in den Maschinenspinnereien brachte wegen der monotonen Beschäftigung in dem noch weichen Knochensystem der Kinder Rückgratverformungen und Beckenverformungen (bei den Mädchen) hervor und wirkte störend auf das Wachstum, weil die natürliche Bewegung als Ausgleich fehlte. Verdauungsstörungen, Anfälligkeit gegen Lungentuberkulose und frühreife Entwicklung waren ferner medizinisch feststellbar. Als schlimmstes Übel erschien es $\mathrm{ihm}$, daß die eintönige mechanische Tätigkeit jede geistige Betätigung verhinderte und Tausende von Kindern vom Schulunterricht fernhielt, der nur auf privater Ebene völlig unzureichend erteilt wurde. Deutsche Statistiken über die Verbreitung des Analphabetentums lassen die großen Mängel des elementaren Bildungswesens deutlich erkennen ${ }^{60}$.

${ }^{59}$ Der Gedanke, daß ohne die Fabriken das Frauen- und Kinderelend noch größer gewesen wäre, ist mehrfach in der englischen Forschung vertreten worden. Bemerkenswerterweise hat sich auch ein deutscher Englandreisender schon 1844 in diesem Sinne geäußert. So meinte der berühmte sächsische Leibarzt und Mitbegründer der modernen Psychologie Carl Gustav Carus nach einer Besichtigung von Fabriken mit Frauen- und Kinderarbeit in Schottland, der Gedanke an die Kinderarbeit habe doch etwas Versöhnendes, wenn man das rastlose Aufkeimen der Menschensaat mit der Beschränktheit der Mittel vergleiche. So erscheine es letztlich als eine Wohltat, daß der menschliche Geist ein Mittel ersonnen habe, massenweise für die Ernährung dieser vielen Kinder zu sorgen und ihnen wenigstens eine Existenz möglich zu machen. Damit sollte die Kinderarbeit selbst natürlich nicht verteidigt werden. Carus nannte sie hart kritisierend einen "pathologischen Auswuchs unserer...kranken Zeit". Vgl. Carus, England und Schottland 1, 341; vgl. P. Deane, The First Industrial Revolution, 1965, 147.

${ }^{60}$ Meidinger, Das Britische Reich in Europa, 491-521. Uber die. mangelnde Erziehung der unteren Volksklassen in England haben sich ferner unter anderem geäußert: C. F. Nebenius, Bemerkungen über den
Nach Friedrich Engels sind 1839 die Hälfte aller Fabrikarbeiter unter 18 Jahren und über drei Viertel Kinder, Frauen und Jugendliche gewesen ${ }^{61}$. Die Zahlen beruhen auf den Angaben eines TorySprechers im Unterhaus und werden von der deutschen Englandliteratur bestätigt. Prüft man dies in der Textilindustrie nach, dann erkennt man, daß unter zwölf Jahren in der Baumwoll- und Leinenindustrie aber nur je $3,7 \mathrm{v} . \mathrm{H}$. und in der Wollindustrie 6,7 v. H. aller Beschäftigten waren ${ }^{62}$. Nur die stark hausgewerblich betriebene Seidenindustrie fällt mit einem hohen Kinderanteil unter zwölf Jahren (20,9 v. H.) aus dem Rahmen. Insgesamt schneidet die am weitesten mechanisierte Baumwollindustrie um $1840 \mathrm{am}$ besten ab. Allerdings hat sie und die Wollindustrie einen hohen Anteil weiblicher Arbeitskräfte ${ }^{63}$. Die Tabellen lassen sich vorsichtig dahin interpretieren, daß um 1840 nicht mehr die

Zustand Großbritanniens in staatswirthschaftlicher Hinsicht, 1818, 82 bis 87; Richter, Bemerkungen, 88; Huber, Erinnerungen, 688-700; J. Venedey, England 3, 1845, 172, 4-9; W. Seyffarth, Europa, seine Länder und Bewohner 2: England und Wales mit ihren Bewohnern, 1851, $395 \mathrm{f}$. $\mathrm{Zu}$ dem negativen Urteil über das englische Bildungs- und Erziehungswesen hat vor allem auch ein englisches Buch beigetragen, dessen Ubersetzung immer wieder zitiert wurde: E. (G. E.) Bulwer (später Lytton Bulwer, 1st Baron of Knebleworth), England und die Engländer. Aus dem Englischen von L. Lax, 2 und 3, 1833.

${ }_{61}$ Engels, Lage der arbeitenden Klasse, 210.

${ }^{62}$ Alter der Arbeitskräfte in der englischen Textilindustrie 1840 in v. $\mathrm{H}$.

\begin{tabular}{|c|c|c|c|c|c|}
\hline Jahre & Baumwolle & Wolle & & Leinen & Seide \\
\hline $8-12$ & 3,7 & 6,7 & & 3,7 & 20,9 \\
\hline $12-13$ & 9,3 & 12,0 & & 12,2 & 8,7 \\
\hline $13-18$ & 29,8 & 29,8 & 1 & 36,1 & 30,8 \\
\hline über 18 & 57,2 & 51,5 & & 48,0 & 39,6 \\
\hline
\end{tabular}

Quelle: Porter, Progress of the Nation, zitiert nach Venedey, England 3, 342.

63 Verhältnis der männlichen und weiblichen Arbeitskräfte in der englischen Textilindustrie 1840 in v. $\mathrm{H}$.

\begin{tabular}{lllll} 
Arbeitskräfte & Baumwolle & Wolle & Leinen & Seide \\
\hline Frauen und Mädchen & 45,7 & 52,5 & 31,2 & 33,2 \\
Männer und Knaben & 54,3 & 47,5 & 68,8 & 66,8
\end{tabular}

Quelle: wie unter Fußnote 62. 
Kinder, sondern der hohe Prozentsatz von weiblichen Jugendlichen das Problem war. Der Höhepunkt der Kinderarbeit war in der Textilindustrie zu Engels Zeiten sicherlich schon längst überschritten. Das größte Übel bei der Kinderarbeit stellte das nichtzentralisierte und nichtmechanisierte Heimgewerbe dar. Das darf freilich nicht darüber hinwegtäuschen, daß die Kinderarbeit noch in anderen Wirtschaftszweigen grassierte, z. B. in den Knopf- und Stecknadelfabriken oder den Steinkohlenzechen. Alle Reisenden betonen, daß aber die Arbeitsplatzbedingungen verschieden seien: Auf der einen Seite fand man Fabrikgebäude neuester Konstruktion mit gut beleuchteten Sälen, Ventilation, Schulunterricht und Spielplätzen (z. B. bei der Mechanischen Flachsspinnerei William Marshall in Leeds), andererseits veraltete Betriebe voller Staub und Hitze, wo die Arbeitenden einen kranken, erschlafften und ausgezehrten Eindruck machten. Anscheinend sind die Zustände in den "Thin Sea Coal Mines“, kleinen, wenig kapitalkräftigen Gruben mit dünnen Kohlenflözen bei Newcastle-upon-Tyne, am schlimmsten gewesen ${ }^{64}$. Nach einem amtlichen Bericht, der auch von der preußischen Regierung übersetzt wurde, hatten Kinder vom sechsten bis zum achten Lebensjahr zehn bis zwölf Stunden lang die Streckentüren vor den anrollenden Kohlenzügen unter Tage zu öffnen und wegen der notwendigen Ventilation wieder $\mathrm{zu}$ schließen und vom 16. bis 18. Lebensjahr im niedrigen Streb von 0,76 m Höhe Kohlenwagen oder Kohlenschlitten zu ziehen oder $\mathrm{zu}$ schieben $^{65}$.

Sicherlich ist es falsch, die Kinderarbeit als vorindustrielles Relikt abzutun, sie nur ökonomisch zu betrachten oder gar optimistisch zu bagatellisieren. Mögliche Übertreibungen auf der einen Seite werden wahrscheinlich durch Vertuschungen auf der anderen Seite kompensiert. Das authentische Beobachtungsmaterial in den deutschen Quellen ist in seiner Masse erdrückend und $\therefore$

64 Kohl, Reisen in England 2, 46.

65 Children's Employment Commission. Appendix to the second Report of the Commissioners: Trade and Manufactures. Part I. Reports and Evidence from Sub-Commissioners. Presented to both Houses of Parliament by Command of Her Majesty, 1842. Vgl. Akten betr. die Beschäftigung jugendlicher Arbeiter in Fabriken, Berg-, Hütten- und Pochwerken und das dafür emanierte Regulativ vom 9.3.1839. Deutsches Zentralarchiv Merseburg Rep. 120 BB Tit. VIII, Nr. 1. deprimierend. Allerdings ist für den Historiker nicht die heutige Bewertung, sondern das damalige Bewußtsein der Menschen darüber entscheidend. Kennzeichnend für die damalige Einstellung ist es, daß Eltern und Unternehmer alles daran setzten, die ersten gesetzlichen Einschränkungen zu umgehen. Die Anstöße zu den Reformen gingen von einigen Philantropen der Mittelklasse sowie den toryistischen Großgrundbesitzern aus, die den liberalen Fabrikanten feindlich gesonnen waren. Die Agitation gegen die Kinderarbeit ist nicht zuletzt auch durch das romantische Zeitgefühl ausgelöst worden, das ein neues Verhältnis zum Kind an sich begründete. Insgesamt weiß man wenig, wie Kinder der Unterschichten außerhalb der Fabriken lebten und arbeiteten. Man kann nur dumpf vermuten, daß auch dort die Kette des Elends für sie nicht abgerissen ist, wie Charles Dickens zeigt ${ }^{66}$. Ein noch völlig ungeschriebenes Kapitel ist z.B. das Schicksal der meist jugendlichen Näherinnen, Puזzmacherinnen, Hemdenmacherinnen usw., von denen es allein in London um 1850 rund 38.000 gab, die nach dem berïchtigten "Schwitzsystem“ (Sweating system) von kleinen habgierigen $\mathrm{Zwischenmeisterinnen} \mathrm{bis} \mathrm{aufs} \mathrm{Blut} \mathrm{ausgepreßt}$ wurden $^{67}$. Die Löhne konnten hier so unerhört tief herabgedrückt werden, weil besonders in der letzten Phase der Frühindustrialisierung Mädchen und junge Frauen in die Städte strömten und sich gegenseitig Konkurrenz machten. Kein Gesetz, keine Zunft und keine Gewerkschaft stärkte ihre Position auf dem Arbeitsmarkt.

Abschließend lassen sich die zeitgenössischen deutschen Betrachtungen über die Rolle des Faktors Arbeit in der britischen Frühindustrialisierung wie folgt generalisieren: Der Arbeitsmarkt hat keineswegs so elastisch reagiert, wie es Wirtschaftstheorien von Adam Smith und Malthus bis zu Marx, Lassalle und Bücher immer wieder dargestellt haben. In Landwirtschaft, Handwerk und Heimgewerbe werden bei empirischer Betrachtung noch eine Fülle von tradierten Privilegien und Sonderarbeitsbeziehungen sichtbar, die eine reine Orientierung am Gesetz von Angebot und Nachfrage

${ }^{66}$ Ch. Dickens, Oliver Twist, 1837-1838; ders., David Copperfield, $1849-1850$.

${ }_{67}$ Vgl. Marx, Das Kapital 1, 263, 3, 115 ff.; Engels, Lage der arbeitenden Klasse, 276-279; F. Lewald, England und Schottland. Reisetagebuch 2, 1852, 543-548; Seyffarth, Europa 1, 101-107. 
durchkreuzen. Vor allem ist es eine historisch unzulässige Simplifizierung, eine direkte Umschichtung von der ländlichen Armut infolge der Einhegungen in die industrielle Lohnarbeiterschaft oder eine direkte Degradierung etwa des Handwebers durch die industrielle Mechanisierung zu konstruieren. Schon vor und beim Beginn des "Take-Off into sustained Growth" befanden sich große Teile der Wirtschaft in einem krisenhaft zugespitzten Wandel ihrer Arbeitsverfassung, was alle Thesen von einem vorherigen „goldenen Zeitalter" zu Legenden stempelt. Es gibt keinen Zweifel, daß zwischen 1750 und 1850 ein kontinuierliches Angebot billiger Arbeitskraft geherrscht hat, was mit den Faktoren Bevölkerungsvermehrung, Verstädterung, irische Einwanderung, technische Innovation und Verlust ländlicher Eígenversorgung zusammen gesehen werden muß. Der Terminus „billige Arbeitskraft“ ist freilich nicht mit niedrigem Lebensstandard identisch, wie Kaufkraftvergleiche mit der vorindustriellen Periode oder mit zeitgenössischen französischen und deutschen Verhältnissen zeigen ${ }^{68}$. Das Vorhandensein eines großen billigen Arbeitskräftereservoirs hat die Unternehmer zusammen mit anderen Faktoren (natürliche Resourcen, Standortlage, Kapitalakkumulation, technischer Fortschritt, liberale Gesetzgebung usw.) zu fortwährenden Investitionen verlockt, da trotz sinkender Preise hohe Gewinne ermöglicht wurden. Gleichzeitig stieg aber dadurch auch die Nachfrage nach hochqualifizierten Arbeitskräften, die nicht im ausreichenden Maße zur Verfügung standen. Alte handwerkliche und neue technische Eliten konnten sich einerseits so einen überdurchschnittlichen ökonomischen wie sozialen Status bewahren oder neu erringen. Inhumane "Exploitation“ der billigen Arbeitskraft ereignete sich andererseits überall dort, wo weder Selbsthilfe noch Staatshilfe die Position auf dem Arbeitsmarkt stärkten.

Die auf Engels zurückgehende ältere Annahme, die Fabrikarbeiter seien „die ältesten Kinder der industriellen Revolution“,

68 Deane, The First Industrial Revolution, 142; B. Hildebrand, Die Nationalökonomie der Gegenwart und Zukunft (1848), in: Sammlung sozialwissenschaftlicher Meister, hrsg. von H. Waentig 22, 1922, $140 \mathrm{ff}$. (Kap. Vergleichung des industriellen Proletariats Englands mit dem deutschen Handwerksproletariat in industriell unterentwickelten Gegenden) und $151 \mathrm{ff}$. (Kap. Vergleichung der britischen Arbeiterzustände der Gegenwart mit denen der Vergangenheit). beruht auf einem rückblickend allerdings verständlichen begrifflichen Mißverständnis, da in den zeitgenössischen Schriften wie auch in der Gesetzgebung meistens nur von den „manufacturing classes" die Rede ist, wobei der Plural bezeichnend ist ${ }^{69}$. In Wahrheit waren die Fabrikarbeiter gerade die letzten und spätesten Kinder des neuen Maschinenzeitalters. Auch das Engelsche Werk handelt fast überwiegend von der städtischen Armut, vom Handwerk und von den Land- und Heimarbeitern, was dann alles unter den Begriff des uniformen Proletariers subsummiert wird. Die daraus abgeleitete These, die frühindustriellen Arbeitskräfte seien überwiegend ungelernt und daher sozial homogen und im Arbeitseinsatz austauschbar gewesen, ist sicherlich in dieser Form nicht richtig: Ganz im Gegenteil hat die mangelnde Anpassungsfähigkeit an die neuen technischen und ökonomischen Innovationen sowie das überkommene Geflecht sozialer Differenzierung den Industrialisierungsprozeß insgesamt verzögert und den vorhandenen älteren Pauperismus unnötig lange perpetuiert.

Wie schon der Marburger Okonom Bruno Hildebrand als einer der subtilsten Kenner der sozialen Frage in der britischen Frühindustrialisierung 1848 in einer fundamentalen Kritik des Engelschen Werkes feststellte, sind viele darin mitgeteilten Einzelheiten richtig, „aber das Ganze ist falsch“. Es stelle nämlich nur die Schattenseiten der neuen Industrie dar. Das sei ebensowenig berechtigt „wie eine Statistik der menschlichen Gesundheit, der bloß Beobachtungen in Krankenhäusern und Hospitälern zu Grunde liegen "70. Engels geht, was von Hildebrand mit zahlreichen statistisch-empirischen Beispielen belegt wird, von manchmal falschen historischen Voraussetzungen bei der Beurteilung damaliger Zustände aus, läßt wesentliche Faktoren aus, während andere

69 Auf die soziale Klassenproblematik und die damit zusammenhängende Begriffsgeschichte kann im begrenzten Rahmen dieser Abhandlung nicht eingegangen werden. Vgl. dazu die immer noch unübertroffene Studie von W. Conze, Vom „Pöbel“ zum „Proletariat“. Sozialgeschichtliche Voraussetzungen für den Sozialismus in Deutschland, in: Moderne deutsche Sozialgeschichte. Hrsg. von H.-U. Wehler, 1966, 111-136.

${ }^{70}$ Hildebrand, Die Nationalökonomie, 138. Vgl. ferner die gedankenreiche kritische Auseinandersetzung mit dem Engelschen Buch bei dem Erlanger Nationalökonomen Ch. Weinlig, Archiv der politischen Oekonomie und Polizeywissenschaft N. F. 3, 1845, 74-98. 
über Gebühr verallgemeinert werden, was dann zu Fehlurteilen führt.

Alle diese Kritik kann gewiß dem mit „sittlicher Wärme“, sprachlicher Brillanz und politischer Verve geschriebenen Engelschen Werk nichts von seiner eminenten historischen Bedeutung nehmen, wurde doch hier erstmals die soziale Seite der Frühindustrialisierung in ihrer ganzen epochalen Bedeutung registriert, was auch einem Hegel, Baader, Mohl, Villermé, de Morogue cder Buret vorher noch nicht gelungen war. Aber auch Engels war nur, wie sein Biograph Gustav Mayer später feinsinnig formulierte, auf den von der Lokomotive Zeitgeist gezogenen Zug gesprungen ${ }^{71}$. So wertvoll sein Buch als sozialgeschichtliches Dokument im Rahmen einer "littérature engagée" bleiben wird, so wenig reicht es heute $\mathrm{zu}$ einer ideologisch entdramatisierten Betrachtung der britischen Frühindustrialisierung aus.

71 Gustav Mayer, Friedrich Engels 1, 1920, 217. Vgl. ferner Hans Pelger (Hrsg.), Friedrich Engels 1820-1970, Schriftenreihe des Forschungsinstituts der Friedrich-Ebert-Stiftung 85, 1971; W. O. Henderson, The Life of Friedrich Engels, 2 Bde., 1974. 